Public Relations

als Machtmanagement 
Jan Lies

\section{Public Relations \\ als Machtmanagement}

Die systemfunktionalistische Synthese der Evolutionsökonomik

Springer Gabler 
Jan Lies

Hamm, Deutschland

Vollständiger Abdruck der von der Universität Witten-Herdecke genehmigten Habilitationsschrift.

ISBN 978-3-8349-4496-2

ISBN 978-3-8349-4497-9 (eBook)

DOI $10.1007 / 978-3-8349-4497-9$

Die Deutsche Nationalbibliothek verzeichnet diese Publikation in der Deutschen Nationalbibliografie; detaillierte bibliografische Daten sind im Internet über http://dnb.d-nb.de abrufbar.

Springer Gabler

(C) Springer Fachmedien Wiesbaden 2012

Das Werk einschließlich aller seiner Teile ist urheberrechtlich geschützt. Jede Verwertung, die nicht ausdrücklich vom Urheberrechtsgesetz zugelassen ist, bedarf der vorherigen $\mathrm{Zu}$ stimmung des Verlags. Das gilt insbesondere für Vervielfältigungen, Bearbeitungen, Übersetzungen, Mikroverfilmungen und die Einspeicherung und Verarbeitung in elektronischen Systemen.

Die Wiedergabe von Gebrauchsnamen, Handelsnamen, Warenbezeichnungen usw. in diesem Werk berechtigt auch ohne besondere Kennzeichnung nicht zu der Annahme, dass solche Namen im Sinne der Warenzeichen- und Markenschutz-Gesetzgebung als frei zu betrachten wären und daher von jedermann benutzt werden dürften.

Gedruckt auf säurefreiem und chlorfrei gebleichtem Papier

Springer Gabler ist eine Marke von Springer DE. Springer DE ist Teil der Fachverlagsgruppe Springer Science+Business Media www.springer-gabler.de 


\section{Zusammenfassung}

Public Relations werden methodologisch in ihren Basiswissenschaften mit zum Teil unvereinbaren Grundannahmen diskutiert. Die oft kollektivistisch denkende Kommunikationswissenschaft als eine der Basiswissenschaften der PR geht im Extremfall wie selbstverständlich von „,der einen“ Öffentlichkeit aus, die als normativ ethisches Handlungskorrektiv Einfluss auf einzelne Organisationen nehmen kann. Dies steht im methodologischen Widerspruch zu den individualistisch denkenden Wirtschaftswissenschaften, die klassisch vom rationalen Denken und Handeln des Einzelnen ausgehen. Mit PR-Diskussionen wie PR als Teil des Marketings, PR als Investor Relations, PR für Marken und andere Themengebiete sind die Wirtschaftswissenschaften als weitere Basiswissenschaft der PR anzusehen. Im Kontrast zwischen Kommunikations- und Wirtschaftswissenschaften werden die polaren Gegensätze der Zugänge zum Thema PR in der jeweiligen Literatur zum Teil als „Akteur vs. System“ besonders deutlich.

Die Suche in der Literatur nach der Funktionsweise im Sinne der Mechanismen- bzw. Institutionenschaffung und -durchsetzung der ethischen Referenzmaßstäbe in der ,pluralistischen Gesellschaft“ verläuft in der Kommunikationswissenschaft oft entlang der Rolle der Medien. Nimmt man an, dass „die Gesellschaft" aus Teilöffentlichkeiten besteht und betrachtet man etwa MarkenCommunities, die Besucher von In-Kneipen oder andere Gesellschaftsgruppen, so stellt sich die Frage, wie sich die Mitglieder einer Teilöffentlichkeit eigentlich finden, zusammenschließen und ihre Einflussnahme organisieren. Die modelltheoretische Diskussion wird umso aktueller, wenn man diese Teilgesellschaften als geschlossene (Sub-)Systeme kennzeichnet, gemäß derer keine gegenseitigen Durchgriffe möglich sind. Wie funktioniert vor diesem Hintergrund PR? Und welchen Mehrwert bietet sie für Organisationen?

Um hierauf Antworten zu finden, sind Public Relations einerseits als Struktur („Beziehungen“), andererseits als Tätigkeit („Management“, „Pressearbeit“) oder aber als Fähigkeit (,soziale Kompetenz") zu unterscheiden - eine Unterscheidung, die in der PR-Diskussion vor allem mit Blick auf den Strukturbegriff zu kurz kommt. Vor diesem Hintergrund erscheint die Substanz des Beziehungsbegriffs als Bestandteil von „Public Relations“ („öffentlichen Beziehungen“) im 
Spannungsfeld der PR-Basiswissenschaften argumentativ noch nicht belastbar zu sein: In vielen PR-Büchern wird er gar nicht systematisch besprochen, sondern ist einfach da. Dabei changiert er zwischen einer Kommunikationsbeziehung mit zwei (personale Kommunikation) oder unendlich vielen (Massenkommunikation) Akteuren in der Kommunikationswissenschaft, reicht über den ,perfekten Tausch“ (Tauschbeziehung, Handelsbeziehung) zwischen zwei Handelspartnern als die klassische Beziehung der Ökonomie über zum Teil dysfunktionale Machtbeziehungen in der Politik, ist ein gesellschaftliches Phänomen vor allem in Form der Struktur zwischen Organisation und ihren Bezugsgruppen und führt bis zum wissens- und kompetenzbezogenen, mulitlateralen Beziehungsgeflecht in der Annäherung von Kulturen auf der Basis psychologischer Prozesse.

Dies zeigt, dass die Psychologie mit Kerndiskussionen wie jener der individuellen und kollektivierten Wahrnehmung und Interpretation ebenso zu den Basiswissenschaften der PR zählt, wie die Soziologie mit der derzeit oft systemtheoretischen Betrachtung teilgesellschaftlicher Strukturen. Dazu können etwa Marken-Communities gerechnet werden, die auf der Basis gemeinschaftlicher psychologischer Prozesse entstehen: Entsprechend hinterfragungsbedürftig ist das viel diskutierte Vertrauenskonstrukt. Auf der Seite der Kommunikationswissenschaft heißt es, dass es im Wesentlichen auf der Basis von Transparenz zu einer Verständigung und/oder Integration führt. Auf der Seite der Wirtschaftswissenschaften wird dagegen im Rahmen von Positionierungsstrategien in der Monopolisierung von Information ein Wettbewerbsvorteil gesehen, der in der Abgrenzung und nicht in der Integration einen Sinn der PR versteht.

An diesen ausgewählten Konfliktthemen aufgezeigt, besteht der Bedarf einer wissenschaftsübergreifenden Modellumgebung für PR, welche die vor allem funktionalistische PR-Diskussion der Wirtschaftswissenschaften mit der oft systemtheoretischen PR-Diskussion aus der Kommunikationswissenschaft respektive der Soziologie zusammenführt: Nicht „Akteur oder System“, sondern „Akteur und System“ lautet die Herausforderung. Ein Angebot hierfür unterbreitet die systemfunktionalistische Synthese, die als evolutionsökonomischer PRAnsatz gekennzeichnet wird.

Grundsätzlich zeigt sich die Evolutionsökonomik mit den Implikationen des VSB-Paradigmas offen für die Erkenntnisse von Nachbarwissenschaften. Zudem findet die Systemtheorie einschlägige Anwendungsmöglichkeiten in der betriebsund kommunikationswissenschaftlichen Diskussion. So knüpft die systemfunk- 
tionalistische Synthese mit Hilfe modifizierter Annahmen der klassischen Ökonomie an die systemtheoretische Diskussion an und konkretisiert das Sozialkapital als wissensbezogene Institution in sozialen Netzwerken, die zum Beziehungsbegriff zurückführen. Als Wissen von Gruppen interpretiert, verleiht es Organisationen einen Vorsprung gegenüber Wettbewerbern in der Realisierungsfähigkeit wertvoller Ereignisse. So sind etwa Marken ein „sozialer Wille“ tendenziell geschlossenener Marken-Communities, die über ein gemeinsames Wissen verfügen. Sie aus Sicht der Community gezielt bedienen zu können, bedeutet im Ergebnis Macht.

Evolutionsprozesse sind so betrachtet dynamische Machtrelationen, die die Mitglieder einer Organisation verlieren, erhalten oder sich neu erschließen. Solche Machtrelationen zu erschließen bzw. zu erhalten, ist eine nicht ohne Weiteres von Dritten kopierbare Kompetenz, die dazu führt, dass diese Organisation das wertvolle Ereignis des Tausches leichter realisiert als ihr Wettbewerber. Dieser Vorsprung, der auf der gemeinschaftlichen Wahrnehmung und Interpretation von Gruppen beruht, wird hier als Sozialkapital verstanden. Ein Kernmodul für diese machttheoretische Theorie liegt in der Sozialpsychologie, welche die PRDiskussionen der eher individuellen Wirtschaftwissenschaften und der eher kollektivierten Kommunikationswissenschaften zusammenführt.

Insgesamt ist festzuhalten, dass die PR-Diskussion systematisch als wissenschaftsübergreifende Debatte anzulegen ist, da PR als Machtmanagement heute sowohl auf wirtschafts-, politik- und kommunikationswissenschaftliche als auch auf soziologische, psychologische und kulturelle Diskussionen zurückgreift. Entsprechend problematisch ist die Tatsache, dass die Managementausbildung oft in der klassischen Betriebswirtschaft verhaftet ist und damit zu kurz greift. Für den Mehrwert von PR und ihre Anwendungskompetenz besteht in Theorie und Praxis ein noch immer ein zu geringes Verständnis. 


\section{Inhaltsverzeichnis}

Zusammenfassung ........................................................................................................ V

Abbildungsverzeichnis.................................................................................... XVII

1 Problemstellung .................................................................................................... 1

1.1 Struktur und Wirkung von Beziehungen............................................... 1

1.2 Unterschiedliche Methodologien der Public Relations ......................... 5

1.3 PR-Theorie: Systemfunktionalistische Synthese …………………..... 7

1.4 Leitfragen der Basiswissenschaften ……………………………….... 8

1.5 Ausgangspunkt der systemfunktionalistischen Synthese _.................... 9

1.6 Zielpunkt der systemfunktionalistischen Synthese ............................. 11

1.7 Vorgehensweise ................................................................................ 13

2 Wirtschaftswissenschaften und Public Relations ................................... 17

2.1 Ausgangspunkt: (Neo-)klassische Ökonomie ……………………....... 20

2.2 PR-Relevante Annahmen aus Klassik/Neoklassik ...............................25

2.3 Die Rolle der PR in der Klassik/Neoklassik ........................................ 29

2.4 Wirtschaftswissenschaftliche PR-Anwendungen................................. 31

2.4.1 Die wohlfahrtsökonomische PR-Diskussion ............................ 31

2.4.2 Die funktionalistische PR-Diskussion ..................................... 33

2.4.3 Die kybernetische PR-Diskussion .............................................. 36

2.4.4 Die marketing-theoretische PR-Diskussion............................... 41

2.4.5 Relationship-Marketing: Beziehungsdiskussion..................... 44

2.4.6 PR als Viral Marketing ........................................................... 47

2.4.7 PR als Markenmanagement ................................................... 48

2.4.8 PR als Stakeholder- und Reputations-Management .............. 52

2.4.9 PR als Media Relations und Investor Relations....................... 57

2.4.10 PR als Risikokommunikation und -management.................... 59

2.4.11 PR als Krisenprävention und -management..............................6 62

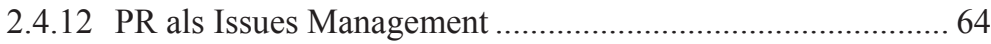

2.4.13 PR als Change Communications …………………………........ 65 
2.4.14 PR als Teil des Innovationsmanagements ............................ 67

2.4.15 PR als interne Kommunikation, Führung, Mikropolitik........ 70

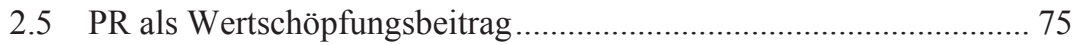

2.6 PR als Klammer für Managementaufgaben ....................................... 78

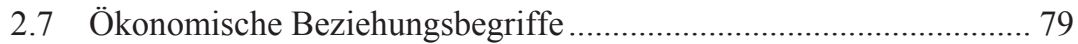

2.7.1 Beziehungen in der klassischen Ökonomie .......................... 80

2.7.2 Beziehungen in der Neuen Institutionenökonomik ............... 81

2.7.3 Beziehungen der Betriebswirtschaft..................................... 83

2.8 Ökonomische Macht- und Beziehungsbegriffe.................................. 88

2.9 Fazit: Modelltheoretische Impulse für die PR-Theorie....................... 91

3 Politikwissenschaft und Public Relations ................................................ 95

3.1 Demokratie und Kommunikationsgebot ............................................. 97

3.2 Steuerung, Kommunikation, Macht ................................................... 97

3.3 Politische Kommunikation und politisches Handeln .......................... 99

3.3.1 Die Betreiber politischer Kommunikation............................ 100

3.3.2 Politische Institutionen als Kommunikationskanäle............. 103

3.3.2.1 PR als Formen politischer Kommunikation ....................... 104

3.3.2.2 PR als Public Affairs und Lobbying................................... 106

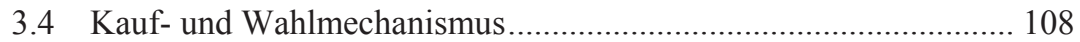

3.4.1 Das politisch-kommunikative Spannungsfeld .................... 109

3.4.2 Öffentliche Hand: Kultur, Image, Kommunikation.............. 112

3.5 Politische Beziehungen und politische Macht ................................. 114

3.5.1 Beziehungsbegriffe in der Politikwissenschaft.................... 114

3.5.2 Politische Macht und Systemtheorie ................................. 116

3.5.2.1 Macht: Öffentlichkeit als Legitimation ............................. 117

3.5.2.2 Weiche Macht und internationale Beziehungen .................. 120

3.6 Fazit: Modelltheoretische Impulse für die PR-Theorie.................... 121

4 Kommunikationswissenschaft und Public Relations ............................. 127

4.1 Publizistik, Kommunikations- und Medienwissenschaft................ 129

4.2 Kommunikationswissenschaftliche PR-Klassik.............................. 130

4.3 Kommunikation als Basis von PR ............................................. 132

4.3.1 Kommunikation als wissenschaftsübergreifender Begriff... 135

4.3.2 Dialog: Kommunikation mit Stil und Haltung .................... 136 
4.3.3 PR zur Verständigung - Burkart......................................... 138

4.4 Handlung und Kommunikation...................................................... 140

4.5 PR und Massenkommunikation ................................................... 143

4.5.1 PR, Öffentlichkeit und öffentliche Meinung ....................... 145

4.5.2 PR, Medien, Realität, Konstruktivismus - Merten .............. 149

4.5.3 PR als Media Relations .................................................... 151

4.6 Aspekte von Kommunikation und Macht ...................................... 153

4.6.1 Determinationsforschung als Machtanalyse ........................ 153

4.6.2 PR und Macht: Foucault - Dorer/Marschik........................ 155

4.6.3 PR als Legitimationsmacht - Ronneberger ....................... 160

4.6.4 PR für öffentliches Vertrauen - Bentele............................. 162

4.7 Beziehungen in der Kommunikationswissenschaft.......................... 164

4.8 Fazit: Modelltheoretische Impulse für die PR-Theorie.................... 166

$5 \quad$ Psychologie und Public Relations ............................................................. 173

5.1 Die (Sozial-)psychologie als Basiswissenschaft der PR ….............. 176

5.1.1 Psychologische Beiträge zur PR-Diskussion....................... 178

5.1.2 Public Relations und Massenpsychologie........................... 179

5.1.3 Von der Massen- zur Gruppenpsychologie ......................... 181

5.2 Psychologische Grundprozesse ..................................................... 182

5.2.1 Psychologie als Basis individuellen Verhaltens .................. 184

5.2.2 Situationen als handlungsrelevante Umgebungsfaktoren .... 186

5.2.3 Gestaltung und Verzerrung fehlender Reize........................ 188

5.2.4 Deutung als handlungsrelevanter Faktor ........................... 189

5.2.5 Mentale Modelle als Determinante von Entscheidungen .... 189

5.2.5.1 Frames als Interaktionsrahmen für mentale Modelle........... 190

5.2.5.2 Kollektivierte mentale Modelle ......................................... 191

5.2.6 Emotionen, Kommunikation und Handlung ........................ 193

5.2.7 Emotion und Motivation................................................... 194

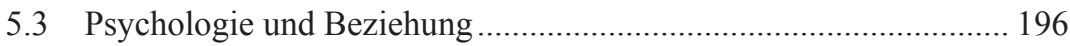

5.3.1 Persönliche und soziale Beziehungen................................. 196

5.3.2 Vier-Ohren-Modell: Kommunikation und Beziehung......... 197

5.3.3 Kommunikation- und Interaktion in der Psychologie........... 198

5.3.4 Entstehung von Beziehungen ........................................... 201 


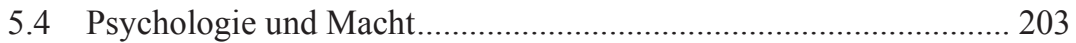

5.4.1 Macht, Identifikation, Charisma....................................... 205

5.4.2 Vertrauen, Reputation und Psychologie .............................. 207

5.4.3 Macht, Marke und Psychologie _.......................................... 213

5.5 Fazit: Modelltheoretische Impulse für die PR-Theorie.................... 217

6 Soziologie und Public Relations.............................................................. 223

6.1 Die Soziologie als Basiswissenschaft von PR ............................... 225

6.2 Klassisch-soziologische PR-Diskussion ......................................... 227

6.3 Beziehungen als soziologisches Analyseinteresse ........................... 231

6.3.1 Soziale Prozesse und soziale Beziehungen......................... 231

6.3.2 Beziehungen: Soziologischer Analyserahmen .................... 234

6.3.3 Soziale Netze, soziologischer Beziehungsbegriff................ 236

6.4 Beziehungen, öffentliche Meinung, Gruppenbildung ...................... 240

6.4.1 Die Suche nach gesellschaftlicher Struktur und Wirkung ... 241

6.4.2 Systemtheoretische PR-Diskussion ..................................... 244

6.4.2.1 PR: Systemtheorie - Ronneberger/Rühl............................. 246

6.4.2.2 PR: Systemtheorie und Anthropologie - Avenarius............ 248

6.4.2.3 PR: Systemtheorie und Innovation - Saxer .......................... 251

6.4.2.4 PR: Systemtheorie und autopoietische Wende - Kussin ..... 254

6.4.2.5 PR: Systemtheorie und organisationales Risiko - Herger ... 261

6.4.2.6 PR: Systemtheorie und Organisationsfunktion - Szyszka... 267

6.4.2.7 Soziologische Markendiskussion ...................................... 274

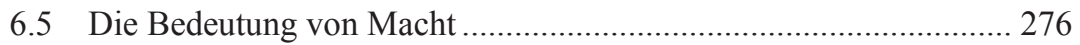

6.5.1 Einfluss, Macht, Zwang, Governance .............................. 279

6.5.2 Geschlossene Netzwerke als Ergebnis von Macht .............. 280

6.6 Fazit: Modelltheoretische Impulse für die PR-Theorie.................... 282

$7 \quad$ Kulturwissenschaften und Public Relations ........................................... 289

7.1 Die Kultur als Basiswissenschaft von PR ……............................... 291

7.2 Die Organisationskultur als Handlungsfeld der PR ......................... 293

7.2.1 Entwicklung der Diskussion um Organisationskultur ......... 293

7.2.2 Kultur-Aspekte in der PR-Diskussion - Hundhausen ......... 297

7.2.3 Wert- und werteorientierte Unternehmensführung.............. 298

7.2.4 PR zur Einflussnahme auf Kultur und Identität..................... 300 
7.2.4.1 Heritage als PR mit Hilfe von Tradition............................. 302

7.2.4.2 Diversity Management als PR für kulturelle Vielfalt .......... 303

7.3 Der kulturwissenschaftliche Beziehungsbegriff: Wissen.................. 306

7.3.1 Internationale PR als Kulturdiskussion .............................. 307

7.3.2 Wissen und Kultur - Analyseraster ..................................... 309

7.3.3 PR als wissensbezogene Anwendungskompetenz ............... 311

7.4 Der kulturwissenschaftliche PR-Ansatz - Faulstich ........................ 313

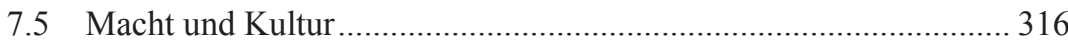

7.6 Fazit: Modelltheoretische Impulse für die PR-Theorie..................... 318

8 Module einer evolutionsökonomischen PR-Theorie .............................. 323

8.1 PR-Blickwinkel und Inkompatibilitäten ........................................ 325

8.2 Systemfunktionalistische Synthese .............................................. 327

8.2.1 Von klassischer Ökonomie zur Evolutionsökonomik.......... 330

8.2.2 Zentrale Erweiterungsaspekte der Evolutionsökonomik ..... 332

8.2.3 Verbindungslinien von PR und Evolutionsökonomik ......... 334

8.3 Modellerweiterungen der klassischen Ökonomie ............................ 338

8.3.1 Das neue St. Galler Management-Modell............................ 340

8.3.2 Die Skandinavische Schule: Märkte als Netzwerke ............ 345

8.3.3 Das sozio-ökonomische Handlungsverständnis.................... 346

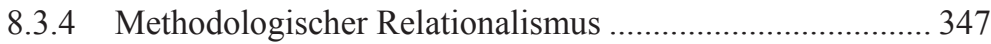

8.3.5 Information, Kommunikation, Wahrnehmung ……............. 348

8.3.6 Externe Effekte als PR-relevante Modellerweiterungen...... 351

8.3.6.1 Typen externer Effekte ...................................................... 352

8.3.6.2 Memetik und konstruktivistisches Management ................. 355

8.3.7 Gruppen als gemeinsame Wissensträger in Netzwerken ..... 357

8.3.7.1 Transaktionskulturen zur Gruppenabgrenzung.................... 357

8.3.7.2 Trends als weiche Faktoren .............................................. 359

8.4 Ordnung als Aufgabe von Public Relations: Dissipation................. 360

8.4.1 Ordnende Energie für ,,mehr Markt“ in Netzwerken............ 361

8.4.2 Ordnungsbildende Prozesse in Systemen ............................ 362

8.5 Konsequenzen für Beziehungen: „Public Relations““....................... 363

8.6 Fazit: Beziehungen als übergreifendes Phänomen............................ 371 
$9 \quad$ PR als evolutionäres Machtmanagement........................................... 373

9.1 Klassische und relationale Macht als Organisationsziel .................. 375

9.1.1 Macht als systemordnendes Managementziel .................... 377

9.1.2 Macht als beziehungsgebundenes Steuerungsmedium ........ 380

9.2 Zur Integration von Macht und Sozialkapital ................................. 385

9.2.1 Sozialkapital - zentrale Positionen ..................................... 386

9.2.2 Sozialkapital und Public Relations .................................... 390

9.2.3 Sozialkapital - offene Fragen .......................................... 392

9.2.3.1 Die grundsätzliche Wirkung von Sozialkapital ................... 394

9.2.3.2 Wann habe ich Sozialkapital? ........................................... 395

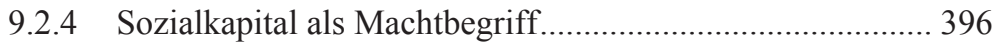

9.2.4.1 Auswirkungen von Netzwerkänderungen auf Macht .......... 397

9.2.4.2 Sozialkapital als privates Gut ............................................. 400

9.2.4.3 Abgeschlossene Netzwerke als Nebenbedingung................ 401

9.2.5 Sozialkapital als Wissensbegriff......................................... 405

9.2.6 Kapitalbegriffe: Sozialkapital und Realkapital................... 406

9.2.6.1 Zur Messbarkeit von Sozialkapital .................................... 409

9.2.6.2 Konzeptionelle Eckpunkte sozialen Kapitals ...................... 412

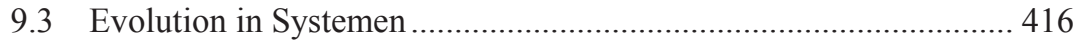

9.3.1 Wahrnehmung als Evolutionselement in Systemen............. 416

9.3.2 Netzwerkdynamik als Wissen über Ordnung ...................... 417

9.3.3 Evolutionsfähigkeit als Kompetenz und Wissen ................. 418

9.3.4 Beispiel: Die Evolution des Download-Musikmarkts ......... 422

9.4 Machtaufbau: Marke, Distinktion, Sozialkapital ............................ 426

9.4.1 PR-Funktionen systemischen Evolutionsmanagements ...... 428

9.4.2 Kompetenzen für das Machtmanagement ......................... 431

9.4.3 PR als multidimensionale Kommunikationskompetenz ...... 435

9.5 Fazit: PR als Machtmanagement ................................................. 437

10 Diskussion: PR neu interpretiert .......................................................... 439

10.1 Klassische PR-Verständnisse in den Basiswissenschaften .............. 440

10.1 .1 „Öffentlichkeit“................................................................. 443

10.1.2 Gesellschaftliche Legitimation und öffentliches Vertrauen 445

10.1.3 „Öffentliche Akzeptanz" als Beziehungsqualität ................ 448

10.2 Geschlossene Systeme, Autopoiesis, Steuerungsskepsis ................. 449 
10.3 Beziehungsaufbau: PR als Medium und System? …....................... 452

10.4 Strong- und Weak-Tie-Netzwerke ................................................ 453

10.5 Die Mikro-, Meso-, Makro-PR-Diskussion .................................... 455

10.6 PR als Dissipationsmanagement .................................................. 456

10.7 Fazit: Antworten auf die Eingangsfragen ....................................... 458

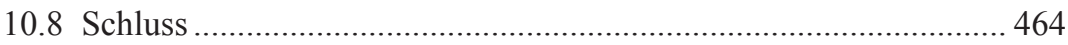

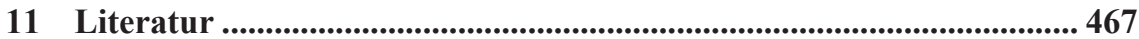

12 Glossar zentraler Begriffe und Ergebnisse ......................................... 491

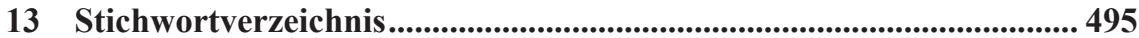




\section{Abbildungsverzeichnis}

Abbildung 1: Leitfragen der PR in den Wissenschaften .................................. 9

Abbildung 2: Architektur der PR-Basiswissenschaften .................................. 13

Abbildung 3: Kommunikationsannahmen der klassischen Ökonomie............. 30

Abbildung 4: Kommunikations-Modelle von Grunig/Hunt ............................ 36

Abbildung 5: Schwerpunkte der Beziehungsqualitätsdiskussion..................... 45

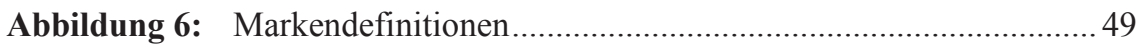

Abbildung 7: Stakeholder-Gruppen und Macht ............................................. 53

Abbildung 8: Perspektiven der Stakeholderdiskussion ................................... 54

Abbildung 9: Folgenorientierter Beziehungsbegriff ..................................... 55

Abbildung 10: Ursachen und Folgen von Risiken .......................................... 60

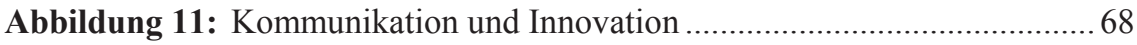

Abbildung 12: PR-Bezüge der Wirtschaftswissenschaften.............................. 76

Abbildung 13: Schwerpunkte von Geschäftsbeziehungen ................................ 85

Abbildung 14: Dimensionen von Geschäftsbeziehungen .............................. 87

Abbildung 15: Wirtschaftswissenschaften: PR-Mehrwerte und Annahmen..... 94

Abbildung 16: Vermittlungsdreieck der Politik .............................................. 98

Abbildung 17: Formelle und informelle Beziehungen .................................. 115

Abbildung 18: Politik zwischen Macht und Öffentlichkeit............................. 119

Abbildung 19: Machtformen................................................................... 121

Abbildung 20: Zentrale Mehrwerte und Annahmen von PR in der Politik..... 125

Abbildung 21: Die Entwicklung des Öffentlichkeitsverständnisses ............... 147

Abbildung 22: Zentrale Mehrwerte und Annahmen von PR in der

Kommunikationswissenschaft .............................................. 171

Abbildung 23: Verhalten von Individuen und Gruppen................................. 183

Abbildung 24: Wahrnehmung von Individuen............................................ 187

Abbildung 25: Kognitive Prozesse der mentalen Modellbildung ................... 191

Abbildung 26: Beispiele für organisationsrelevante Emotionen...................... 194

Abbildung 27: Das vier-Ohren-Modell von Schulz von Thun........................ 198

Abbildung 28: Stadien der Beziehungsbildung........................................... 202

Abbildung 29: Modell der Beziehungsentwicklung...................................... 203

Abbildung 30: Differentielle Vertrauenstheorie ...........................................209 
Abbildung 31: Grundlagen des Vertrauens im Wandel der Zeit.................... 211

Abbildung 32: Dimensionen von Beziehungen .......................................... 214

Abbildung 33: Analyse von Markenbeziehungen........................................ 215

Abbildung 34: Zentrale Mehrwerte und Annahmen von PR in der

Psychologie....................................................................... 222

Abbildung 35: Kennzeichen persönlicher Beziehungen ................................ 234

Abbildung 36: Analyserahmen persönlicher Beziehungen ........................... 235

Abbildung 37: Minimalausstattung eines Netzwerkes.................................. 238

Abbildung 38: Entwicklung von PR in der Gesellschaft ............................... 252

Abbildung 39: Das System der Organisationskommunikation ....................... 264

Abbildung 40: Strategien auf der Grundlagen der Organisations-Matrix ....... 265

Abbildung 41: Öffentliche Kommunikation und Meinungsmärkte ................ 271

Abbildung 42: Modell funktionaler Transparenz.......................................... 274

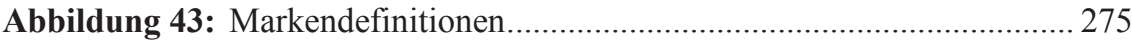

Abbildung 44: Systemtheoretische PR-Beiträge in der Übersicht ................... 283

Abbildung 45: Zentrale Mehrwerte und Annahmen von PR

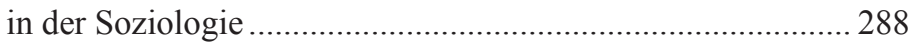

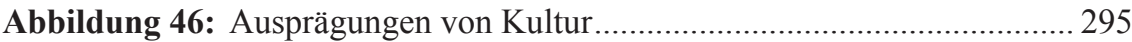

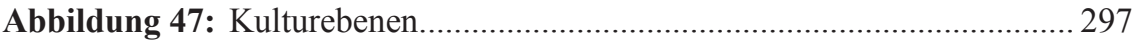

Abbildung 48: Internationale Kommunikation ........................................... 308

Abbildung 49: Analyseraster internationaler Kommunikation ....................... 310

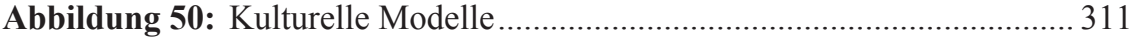

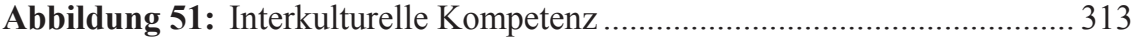

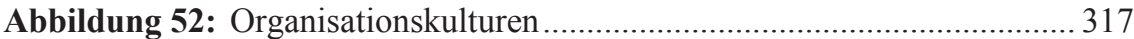

Abbildung 53: Zentrale Mehrwerte und Annahmen von PR in den

Kulturwissenschaften

Abbildung 54: Zentrale Aspekte der systemfunktionalistischen Synthese ..... 329

Abbildung 55: Evolution biologischer und sozio-ökonomischer Systeme ..... 332

Abbildung 56: Vergleich zentraler Annahmen von Klassik und

Evolutionsökonomik

Abbildung 57: Wirtschaftswissenschaftliche Modellerweiterungen für eine machttheoretische PR-Theorie .......................................... 340

Abbildung 58: Das neue St. Galler Management-Modell................................ 343 
Abbildung 59: Kommunikationsfelder zwischen Akteuren eines

Netzwerkes als Voraussetzung von Transaktion

Abbildung 60: Individuelle Informations-/Wahrnehmungsfelder der

Akteure eines Netzwerkes....................................................350

Abbildung 61: Der externe Effekt einer Transaktion...................................... 352

Abbildung 62: Sichtweisen von Beziehungen .............................................. 364

Abbildung 63: Zentrale Aspekte von Beziehungsbegriffen ........................... 369

Abbildung 64: Modifizierte Annahmen der systemfunktionalistischen

Synthese im Vergleich zur klassischen Ökonomie .................. 370

Abbildung 65: Unterschiede von Machtbegriffen.......................................... 382

Abbildung 66: Systemfunktionalistische Synthese - Konstitution der

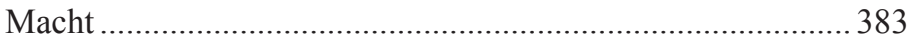

Abbildung 67: PR und Sozialkapital - zentrale Aspekte ………..................... 392

Abbildung 68: Sozialkapital - zentrale Positionen ......................................... 394

Abbildung 69: Machtrelevante Netzwerkelemente......................................... 399

Abbildung 70: Kriterien gruppenbezogener Güter....................................... 402

Abbildung 71: Die relevanten Wissensdimensionen sozialen Kapitals .......... 406

Abbildung 72: Zentrale Definitionen sozialen Kapitals................................ 415

Abbildung 73: Zusammenhänge integrierten Innovationsmanagements ........ 420

Abbildung 74: Marktveränderungen als soziales Drama ............................... 424

Abbildung 75: Aufbau von Machtpositionen in Communities ....................... 427

Abbildung 76: PR-Disziplinen als relationales Machtmanagement................ 429

Abbildung 77: PR-Instrumente im systemischen Evolutionsmanagement ..... 431

Abbildung 78: Institutionalisierung von Macht als Verhandlungsprozess...... 433

Abbildung 79: Kompetenzen für innerbetriebliche Machtrelationen.............. 435

Abbildung 80: Diskussionen in den Basiswissenschaften ............................. 442

Abbildung 81: Strong und weak Ties im Vergleich...................................... 454

Abbildung 82: Merkmale der Stromversorgung und ihre Übertragung auf das Dissipationsmanagement 